\title{
Pancreatic rest in a young woman: a rare cause of dyspepsia
}

\begin{abstract}
Ectopic pancreatic tissue or heterotopic pancreatic tissue, also known as a pancreatic rest, is an uncommon congenital anomaly defined as displacement of small amount of pancreas during embryonic development, resulting in the formation of a nodule located far from the pancreas and without any connection via vascular or anatomical means. It can be seen in any part of the gastrointestinal tract with larger percentages found in stomach followed by small intestine especially in duodenum and jejunum. It may remain silent over period of years or present clinically mimicking symptoms of acid peptic disease, ulcer, upper gastrointestinal tract bleeding and obstruction. Infrequently, it may lead to pancreatitis or pancreatic cancer. Radiological appearances are variable ranging from small lesion to large masses. Histopathological diagnosis remains the gold standard. This report describes the clinical scenario of severe dyspeptic symptoms of twelve weeks duration in a young female. She underwent upper gastroscopic examination, which revealed a nodular lesion in the antral portion of the stomach. After resection of nodule and proton pump inhibitors of 1-month duration her symptoms improved. The histological specimen revealed the presence of pancreatic rest and no evidence of malignancy was noted. The patient is symptom-free and has been followed up in our clinic.
\end{abstract}

Keywords: heterotropic pancreatic tissue, acid peptic disease, pancreatic rest, pancreatitis
Volume 12 Issue 3 - 2021

\author{
Richmond Ronald Gomes \\ Department of Medicine,Ad-din Women's Medical College \\ Hospital, Bangladesh
}

Correspondence: Richmond Ronald Gomes, Associate Professor, Medicine Ad-din Women's Medical College Hospital, Dhaka, Bangladesh, Email rrichi.dmc.k56@gmail.com

Received: May II, 202I | Published: June 08, 202 I

\section{Introduction}

The pancreatic rest is an uncommon congenital anomaly. The incidence in autopsy series varies from 1 to $2 \%$ (range 0.55 to $13 \%$ ). Such tissue may occur throughout the gastrointestinal tract but has a propensity to affect the stomach and the proximal small intestine. Histological features of acinar formation, development of ducts and independent blood supply are usually present. Accordingly four types have been described, type I having more resemblance to normal pancreatic tissue and type IV showing only islets cells. Although the cause of pancreatic rest remains unclear, several theories, including the 'theory of metaplasia,' the 'theory of misplacement' and the latest addition, the 'theory of abnormalities of notch signaling', have been proposed to explain the pathogenesis and occurrence of pancreatic heterotopia. While majority of patients with pancreatic ectopic tissue are asymptomatic, a variety of symptoms are attributed to this ectopic tissue. Depending upon location and bulk of the pancreatic rest the symptoms may include, gastrointestinal bleeding, gastric outlet obstruction, gastric ulceration, pancreatitis and even obstructive jaundice, when located near Ampulla of vater have been described. There are reports when such lesions have presented with acute perforation, what may reflect pancreatitis of stomach and duodenum. When discovered during endoscopic examination it is very important to differentiate these lesions from submucosal tumors and appropriate surgery may be required to confirm the lesion when results of fine needle aspiration are inconclusive. Despite the advent of novel diagnostic modalities, including endoscopic ultrasonography (EUS), computed tomography (CT) and even EUS-guided fine-needle aspiration (EUS-FNA), the differentiation from a neoplasm at times remains a clinical challenge. When in a given case FNAC has proved it to be pancreatic rest and the patient is asymptomatic, such lesions can be monitored. Endoscopic features of this rare entity and a brief review is presented in this report.

\section{Case report}

A 27-year-old female presented with a history of upper abdominal pain, nausea and off and on vomiting of 12 weeks duration. The pain had an intensity of 7/10 and was a burning type of pain with no reference or radiation. She stated that for the previous 2 weeks her pain was aggravated by food intake. She denied any alarming symptoms in the form of loss of appetite and weight loss. There was no history of hematemesis or melena. She had no history of NSAID intake or tuberculosis contact. Her past medical records showed an appendicectomy eight years back. Her clinical examination was normal apart from mild epigastric tenderness and on evaluation, her hemoglobin levels were $14 \mathrm{~g} / \mathrm{dl}$. She had a normal leukocyte count, and liver function and renal function were normal. Serum lipase and amylase levels were not elevated. An ultrasound of her abdomen did not indicate the presence of gallstones and all other organs were normal. She underwent upper gastrointestinal (GI) endoscopy to rule out a gastric ulcer due to the pain being aggravated by food. An upper gastroscopic examination revealed normal esophagus. There was 4 $\mathrm{cm} \times 3 \mathrm{~cm}$ bulge in the antral portion of her stomach, and the overlying mucosa was erythematosus (Figure 1). The fundus and body of the stomach were normal. There was no evidence of duodenal ulcer or growth. Rapid urease test for Helicobacter pylori was negative.A biopsy from the antral portion revealed chronic gastritis, but there was no evidence of Helicobacter pylori. The differential diagnosis of gastrointestinal tumor (GIST) was considered and resection of the nodule and biopsy was performed after color Doppler imaging ruled out the presence of vascular structures along the needle track. However, the microscopic examination (Figure 2) showed islands of pancreatic acini and ducts in the muscularispropria of the stomach. Several dilated ducts were seen extending through all the layers of the muscularispropria. There was evidence of fibrotic reaction and scant mixed inflammatory infiltrate was observed in the area. There was no evidence of malignancy. 
She was prescribed oral proton pump inhibitors $20 \mathrm{mg}$ once daily for 1-month duration only, is now pain-free. She has been followed up by the clinic for the last 6months.

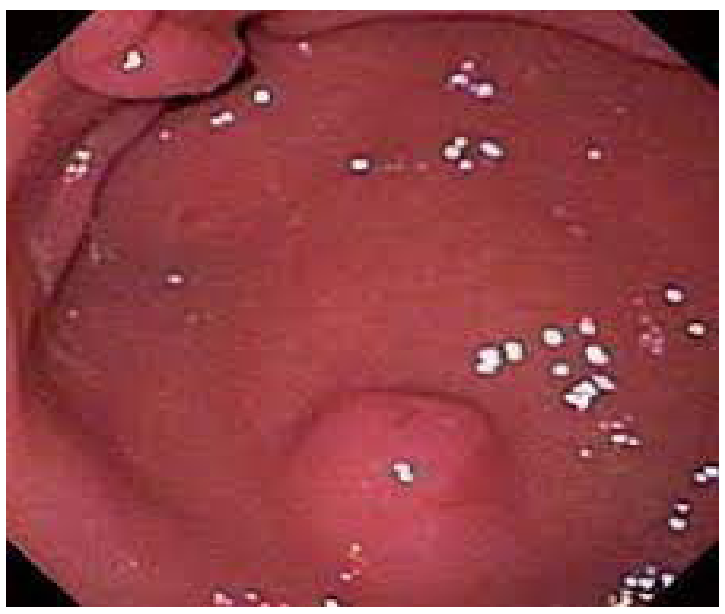

Figure I Endoscopy of upper GIT revealing nodule over gastric antrum.



Figure $\mathbf{2}$ histopathology of the resected nodule showing islands of pancreatic acini.

\section{Discussion}

The results of a recent meta-analysis showed the overall pooled prevalence of non-investigated dyspepsia was $21 \% .{ }^{1}$ The index case had classical dyspeptic symptoms, which are common worldwide, but as there was no response to conventional treatment, an upper gastroscopy was undertaken to rule out any other cause of her symptoms. It was found that she had an antral lesion which proved to be pancreatic rest. Her symptoms completely obviated after the removal of pancreatic rest

Pancreatic rest or ectopic pancreas is a rare disorder. Ectopic pancreatic tissue or heterotopic pancreatic tissue refers to the presence of pancreatic tissue in the submucosal, muscularis or subserosal layers of the gastrointestinal tract outside the normal confines of the pancreas. There is no anatomical or vascular connection with the main pancreas2. It can be named as aberrant, accessory pancreas, or sometimes called as pancreatic choristoma and adenomyoma. It is a congenital abnormality, with a male to female ratio of $3: 13$. It is difficult to determine the true incidence as usually patients are asymptomatic and it is detected on autopsy. A prevalence rate of $0.5 \%-13.7 \%$ is quoted in autopsy results, $0.2 \%$ in upper abdominal surgeries as incidental finding and $0.9 \%$ of gastrectomies. ${ }^{3,4}$ Although it can be found anywhere in the foregut and the proximal-midgut, the most common site is the stomach. ${ }^{5}$ Small percentages include esophagus, ileum, biliary tree, mesentery, and occasionally spleen6. Imaging plays role in diagnosis of symptomatic cases, CT being the investigation of choice.

The first description of this condition was given by Hunt and Bonesteel in 1727 who described it as being present in a diverticulum of the ileum. ${ }^{7}$ Many reports with different presentations have been described in the literature since then. The most common computed tomographic appearance of heterotopic pancreas is usually a small oval intramural mass with microlobulated margins or cystic lesion. The attenuation and enhancement characteristics of these lesions parallel their histologic composition. Acinus-dominant lesions demonstrate avid homogeneous enhancement after intravenous contrast material administration, whereas duct-dominant lesions are hypo vascular and heterogeneous 4 . With the advent of EUS combined with FNA, the overall diagnosis of submucosal gastric lesions have advanced. ${ }^{8}$ The endoscopic differential diagnosis was GIST but FNAC ruled it out. EUS features of pancreatic rest usually correlate with specific histologic components. Often a heterogeneous acoustic image and the sonographic layer of origin of the lesion are observed. ${ }^{9}$ The endoscopic appearance of pancreatic rest is usually that of a firm, slightly irregular nodule in the stomach or elsewhere in the GI tract usually with central umbilication but cystic appearance on EUS has also been reported. ${ }^{10}$ The size of the nodules may vary from 0.2 to $4.0 \mathrm{~cm}$ in diameter. The mucosa over the nodule may be smooth or have a central depression and ducts may empty into the lumen at this site. The other differentials of this endoscopic appearance include a stromal cell tumor, lymphoma, carcinoid, and adenomatous polyp. ${ }^{5}$

Pancreatic rests present with nonspecific gastrointestinal symptoms like epigastric pain and dyspepsia or else it may present with the clinical findings of obstructive jaundice or intestinal obstruction or it may even mimic gastrointestinal system cancer. ${ }^{11}$ The location of the pancreatic rest markedly determines the clinical presentation in a given case. Gunjaca et al. have described pancreatic rest to be the cause of acute abdomen. They described acute abdomen in a patient who was operated due to duodenal perforation following acute inflammation of the pancreatic rest in the duodenal area. ${ }^{12}$ In another clinical scenario of pancreatic rest Flippo et al. described pancreatic rest in the area of ampulla of Vater in a 69-year-old female who had presented with painless obstructive jaundice. ${ }^{13}$ Reports of pseudocyst formation due to this congenital anomaly have also been described in the literature. ${ }^{14}$ On the other hand, Monedero et al. described intussusceptions due to pancreatic rest presenting as a mass into an inverted Meckel's diverticulum. ${ }^{15}$ In yet another clinical scenario, Ugur Kantar et al. reported pancreatic rest in the duodenum to be the cause of intractable diarrhea and authors reported complete resolution of symptoms following resection, as we report in the present case. ${ }^{16}$ At times, patients can present with potential complications of heterotopic pancreas which include pancreatitis, pseudocyst formation, malignant degeneration. ${ }^{17}$ Certain complications may be erroneously diagnosed as malignancy. Although rare, malignant transformation must be considered when submucosal lesions are identified in the stomach. In order to prove that a malignancy arose from ectopic pancreas, three criteria must be fulfilled, according to Guillou, the tumor must be within or near the ectopic pancreatic tissue; a direct transition between pancreatic structures and carcinoma must be observed; and the nonneoplastic pancreatic tissue must, at a minimum, comprise fully developed acini and ductal structures. There are three precursor lesions that give rise to pancreatic cancer: mucinous cystic neoplasm 
(MCN), intraductal papillary mucinous neoplasm (IPMN), and pancreatic intraepithelial neoplasia $(\mathrm{PanIN}) \cdot{ }^{18} \mathrm{It}$ is often impossible to distinguish gastric heterotopic pancreas from primary or metastatic cancer because endoscopic biopsies are often unremarkable. Therefore, frozen sections should be taken rapidly and routinely so as to confirm the diagnosis and avoid unwanted radical surgery such as Whipple's procedure or subtotal gastrectomy. Medical treatment is not effective for heterotopic pancreas.It is managed either endoscopically or surgically depending on the location and size of the lesion. ${ }^{19,20}$

\section{Conclusion}

The present case highlights that pancreatic rest is an unusual cause of dyspepsia and underlines the importance of endoscopic examination in a young patient, irrespective of alarm symptoms, after failure of proton pump therapy. Pancreatic heterotopia is a condition which may give deceptive appearances of a gastrointestinal tract tumor especially stomach. Clinician and radiologist should keep it as differential possibility down the line as recognition of imaging features helps in differentiating it from neoplastic conditions and thus in avoiding unnecessary surgery.

\section{Acknowledgments}

None.

\section{Conflicts of interest}

We, the authors declare that we have no conflicting interests.

\section{Funding}

The authors did not receive any grant for this research study.

\section{References}

1. Lovell RM, Ford AC. Global prevalence of and risk factors for irritable bowel syndrome: A meta-analysis. Clin Gastroenterol Hepatol. 2012;10:712-21.e4.

2. Brian Gilcrease Garcia, Radswiki. Ectopic pancreatic tissue. 2018.

3. Wei R, Wang QB, Chen QH, et al. Upper gastrointestinal tract heterotopic pancreas: findings from $\mathrm{CT}$ and endoscopic imaging with histopathologic correlation. Clin Imaging. 2011;35(5):353-359.

4. Kung JW, Brown A, Kruskal JB, et al. Heterotopic pancreas: typical and atypical imaging findings. Clin Radiol. 2010;65(5):403-407.

5. Kaneda M, Yano T, Yamamoto $T$, et al. Ectopic pancreas in the stomach presenting as an inflammatory abdominal mass. Am $J$ Gastroenterol. 1989;84:663-666.
6. Park SH, Han JK, Choi BI, et al. Heterotopic pancreas of the stomach: CT findings correlated with pathologic findings in six patients. Abdom Imaging. 25(2):119-123.

7. Pearson S. Aberrant pancreas. Review of the literature and report of three cases, one of which produced common and pancreatic duct obstruction. AMA Arch Surg. 1951;63(2):68-186.

8. Matsui M, Goto H, Niwa Y, et al. Preliminary results of fine needle aspiration biopsy histology in upper gastrointestinal submucosal tumors. Endoscopy. 1998;30(9):750-755.

9. Matsushita M, Hajiro K, Okazaki K, et al. Gastric aberrant pancreas: EUS analysis in comparison with the histology. Gastrointest Endosc. 1999;49(4 Pt 1):493-497.

10. Riyaz A, Cohen H. Ectopic pancreas presenting as a submucosal gastric antral tumor that was cystic on EUS. Gastrointest Endosc. 2001;53(6):675677.

11. Shah A, Gordon AR, Ginsberg GG, et al. Case report: Ectopic pancreatic rest in the proximal stomach mimicking gastric neoplasms. Clin Radiol. 2007;62(6):600-602.

12. Gunjaca I, Mlinac-Lucijanic M, Pavlovic A, et al. Inflammation of ectopic pancreatic tissue as unusual cause of duodenal perforation - A case report. Coll Antropol. 2010;34(3):1119-1122.

13. Filippou DK, Vezakis A, Filippou G, et al. A rare case of ectopic pancreas in the ampulla of Vater presented with obstructive jaundice. Ann Ital Chir. 2006;77:517-519.

14. Mulholland KC, Wallace WD, Epanomeritakis E, et al. Pseudocyst formation in gastric ectopic pancreas. JOP. 2004;5(6):498-501.

15. Monedero MD, Ripollés T, Nicolau MJ, et al. Pancreatic pseudotumor in Meckel diverticulum. Abdom Imaging. 2006;31(6):688-690.

16. Ugur Kantar F, Akarsu M, Atilla K, et al. Ectopic pancreas presenting with intractable diarrhea: Case report. Turk J Gastroenterol. 2011;22(4):426429.

17. Lai EC, Tompkins RK. Heterotopic pancreas: review of a 26 year experience. Am J Surg. 1986;151(6):697-700.

18. Sadeghi NR, Godambe A, Shienbaum AJ, et al. Premalignant Gastric Heterotopic Pancreas. Gastroenterol Hepatol Journal (N Y). 2008;4(3):218-221.

19. Kim KJ. Endoscopic resection of ectopic pancreas in the minor papilla. Endoscopy. 2009;41(Suppl 2):E12.

20. Barrocas A, Fontenelle LJ, Williams MJ. Gastric heterotopic pancreas: A case report and review of literature. Am Surg. 1973;39:361-365. 